\title{
Methodology for the Canadian Activase for Stroke Effectiveness Study (CASES)
}

\author{
Michael D. Hill, Alastair M. Buchan and the CASES Investigators
}

\begin{abstract}
Background: Intravenous recombinant tissue plasminogen activator (tPA, alteplase) was conditionally licensed for the treatment of acute ischemic stroke (AIS) in Canada on February 17, 1999. As a condition of licensure, the Canadian Activase for Stroke Effectiveness Study (CASES) was established to monitor the use of alteplase for AIS in Canada. The study involves multiple stakeholders. Methods: CASES is a prospective registry of patients treated with alteplase for AIS. The purposes of this registry are to ensure the safety of the drug in the Canadian context, to assess effectiveness of alteplase for AIS and to gather further information to try to establish which patients are most likely to benefit from treatment. Results: Both community $(n=25)$ and tertiary centres $(n=35)$ have enrolled a total of 944 patients to date. Early results suggest that thrombolytic stroke treatment is both safe and effective among these centres. Conclusions: This paper outlines the development of and methods for the CASES study. The study is an example of a multi-stakeholder collaboration to advance the care of patients with acute stroke.
\end{abstract}

RÉSUMÉ: Méthodologie de l'Étude Canadienne sur l'efficacité d'activase dans le traitement de l'accident vasculaire cérébral (CASES). Introduction: L'activateur du plasminogène tissulaire recombinant (tPA, alteplase) administré par voie intraveineuse a été approuvé sous condition pour le traitement de l'accident vasculaire cérébral ischémique (AVCI) au Canada le 17 février 1999. L'Étude Canadienne sur l'efficacité d'activase dans le traitement de l'AVCI a été établie pour surveiller l'utilisation de l'alteplase dans le traitement de l'AVCI aigu afin de satisfaire à une condition de l'approbation. L'étude implique plusieurs partenaires. Méthodes: CASES est un registre prospectif de patients traités par l'alteplase pour un AVCI aigu. Les buts de ce registre sont de s'assurer de la sécurité du médicament dans le contexte Canadien, d'évaluer l'efficacité de l'alteplase dans le traitement de l'AVCI aigu et d'amasser plus d'informations pour déterminer quels patients sont susceptibles de bénéficier davantage de ce traitement. Résultats: Des centres de soins communautaires $(n=25)$ et tertiaires $(n=35)$ ont enregistré au total 944 patients à date. Les résultats préliminaires suggèrent que le traitement thrombolytique de l'AVC est sécuritaire et efficace dans ces centres. Conclusions: Cet article décrit le développement et les méthodes de l'étude CASES. Cette étude est un exemple d'une collaboration de plusieurs partenaires pour l'avancement des soins chez des patients atteints d'AVC aigu.

Can. J. Neurol. Sci. 2001; 28: 232-238

Although several randomised, controlled trials have now been conducted using intravenous tissue plasminogen activator (tPA, alteplase, Activase ${ }^{\circledR}$ ) for acute ischemic stroke (AIS), only the National Institute of Neurological Disorders and Stroke (NINDS) tPA Stroke Trial which examined a 0-3 hour treatment window, has shown a positive result in the intention-to-treat analysis. ${ }^{1}$ Two European trials, European Co-operative Acute Stroke Study (ECASS)-1 and ECASS-2 examined a 0-6 hour treatment window and failed to demonstrate efficacy in the intention-to-treat analysis of the primary hypothesis. ${ }^{2,3}$ A second North American trial, the Alteplase Thrombolysis for Acute Noninterventional Therapy in Ischemic Stroke - Part B trial, examined the 3-5 hour treatment window and also showed a neutral result. ${ }^{4}$
The differences among these trials have been hotly debated. ${ }^{5-9}$ The Cochrane Stroke Review Group has pooled individual patient data from these four and earlier pilot studies for the 0-6 hour treatment window and demonstrated a benefit for intravenous alteplase therapy. The odds ratio of death or dependence in the alteplase treated vs. untreated group is 0.79

From the Department of Clinical Neurosciences, University of Calgary, Foothills Medical Centre, Seaman Family MR Centre, Calgary, AB, Canada. ReCEIVEDJuly 7, 2000. ACCEPTED INFINALFORM MAY 15, 2001 Reprint requests to: M.D. Hill, University of Calgary, Department of Clinical Neurosciences, Foothills Medical Centre, MRG005 - Seaman Family MR Centre, 1403 29th Street NW, Calgary, AB T2N 2T9 Canada. 
(95\% CI 0.68-0.92) (Figure 1). No effect was observed on total mortality and the treatment effect is even more marked for the 0 3 hour cohort. ${ }^{10}$

The treatment of AIS with intravenous alteplase was licensed by the Food and Drug Administration in the US in 1996 and by the Therapeutic Products Program in Canada in February 1999. ${ }^{11}$ Full approval has not been achieved outside of North America. There are wide intra- and inter-provincial variations in stroke care in Canada and the public is not educated about stroke signs and symptoms. ${ }^{12}$ Thus, the February 1999 licensure of alteplase in Canada was conditional upon the establishment of a study to monitor outcomes. The Canadian Activase for Stroke Effectiveness Study (CASES) was launched as a collaborative effort among the Canadian Stroke Consortium (CSC), the Canadian Stroke Society, the Heart and Stroke Foundation of Canada, Hoffmann-La Roche Canada Ltd., and physicians coastto-coast. The recently funded Canadian Stroke Network has also joined as a partner. The study is a prospective registry of all patients treated with alteplase for AIS beginning February 17, 1999 through June 30, 2001.

\section{Relationships and study organisation}

With a Health Canada mandate to perform a post-marketing registry of alteplase treatment of stroke, Hoffmann-La Roche Canada Ltd., partnered with the CSC Research Committee to launch CASES. The Canadian Stroke Society and the Heart and Stroke Foundation of Canada have been in-kind supporters. The Canadian Stroke Network, a national centres of excellence program, and the CSC have provided administrative funds for the management of the CASES project. Hoffmann-La Roche Canada Ltd has supported the study by providing nominal per patient enrollment honouraria, by supporting stroke via pharmaceutical representatives and by providing educational grants for local and national stroke symposia.

The study is monitored by a steering committee based at the
University of Calgary. A safety committee has been nominated but has not been required to meet.

\section{Methodology}

\section{Study design}

CASES is a prospective observational cohort study. All patients treated with intravenous alteplase for AIS from Feb 17, 1999 through June 30, 2001 are eligible to be included. The study includes 60 active centres in Canada.

\section{Objectives}

The purposes of this study are several fold: (1) to assess the safety of alteplase for stroke in the context of routine care in Canada; (2) to assess whether efficacy demonstrated in randomised clinical trials can be translated into effectiveness in the Canadian case - ie. to assess effectiveness; (3) to gather further information on patients treated with alteplase for AIS to develop a model predictive of outcome; (4) to assess the potential value of a scoring system for acute CT scan changes (the ASPECT score) in predicting outcome after alteplase therapy for AIS.

A fifth objective, the assessment of appropriateness, was initially pre-specified but has been dropped because an assessment of appropriateness will be part of the final multivariable analysis.

\section{Centre and patient eligibility}

All centres that have, or are planning to treat AIS patients with alteplase are eligible to register. In an effort to be comprehensive, the registry will accept information on any patient treated with intravenous alteplase for AIS. Centres are strongly recommended to have a standing acute stroke protocol that conforms to accepted guidelines, ${ }^{1,13}$ to have emergent CT scanning available 24 hours a day and physicians with expertise

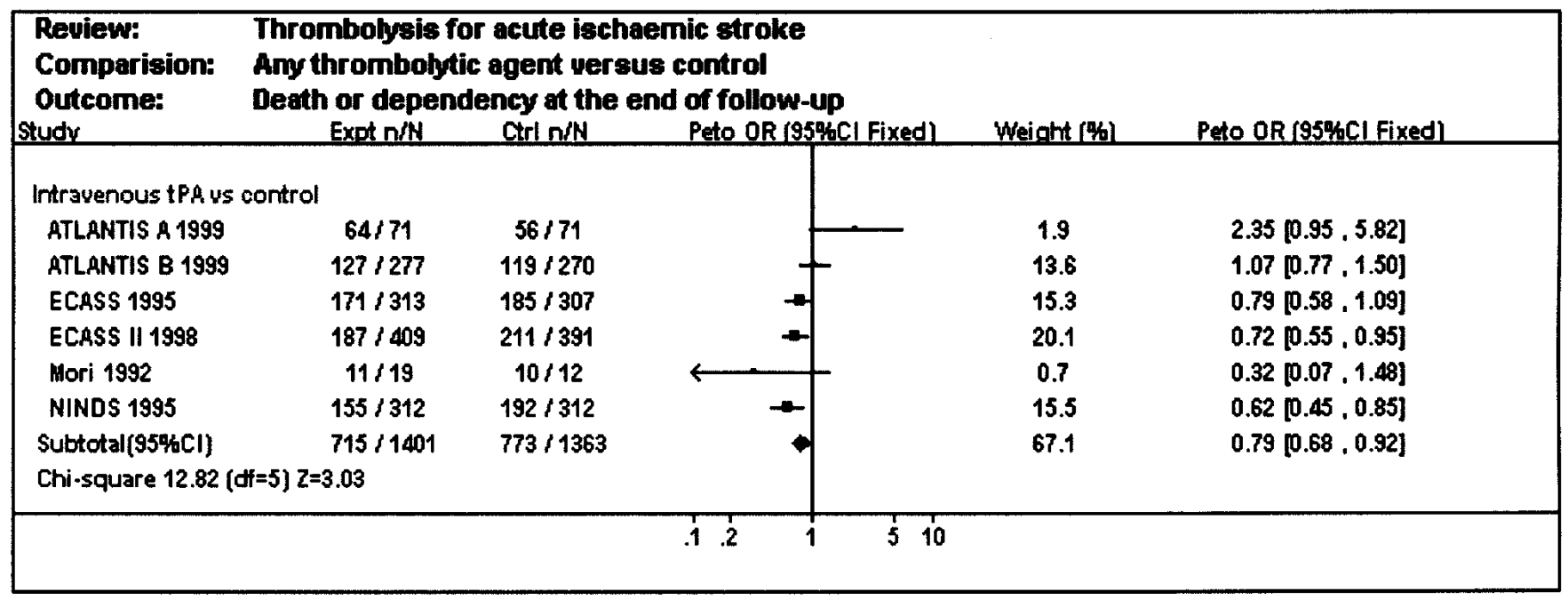

Figure 1: Meta-analysis of data from random controlled trials of Stroke thrombolysis. $n / N=(n)$ number; $(N)$ Total; OR = odds ratio

Modified from the Cochrane Collaboration Stroke Review. Reprinted with permission from Dr. J. Warlaw and Update Software. Adapted from the Cochrane Database of Systematic Reviews. In the Cochrance Library, Issue \#4, 1999. Oxford: Update Software. Updated quarterly. www.cochranelibrary.com-

http://www.update-software.com/about/about-frame.html 
in stroke care available to be on call for acute stroke. Physicianinvestigators do not necessarily have to be neurologists.

\section{Sub-studies}

Two smaller sub-studies within CASES will examine the reasons that preclude treatment among patients who arrive within the three-hour window but are not treated and why some physicians choose intra-arterial rather than intravenous alteplase.

\section{Outcome measures}

Two primary outcome measures, recorded at 90 days, include the modified Rankin (mRS) scale score and the National Institutes of Health Stroke Scale (NIHSS) score. The mRS will be dichotomised into independence (mRS 0-2) vs. dependence/ death (mRS 3-6) as the primary binary outcome. The NIHSS score dichotomised into no/minimal neurological deficit on examination (NIHSS 0-1) vs. neurological deficit (NIHSS > 1) will serve as a second primary outcome variable. Mortality will be analysed by assigning patients the worst score on each scale $(\mathrm{mRS}=6$, NIHSS $=42$ ) for death within the 90-day follow-up period.

Safety outcomes will consist of the proportion of patients suffering any major adverse event. These include symptomatic intracerebral hemorrhage (ICH), orolingual angioedema, anaphylaxis, serious systemic bleeding or any other event resulting in harm to the patient, as judged by the local investigator to be related to the treatment. Symptomatic ICH is defined as any clinically important decline in neurological status that occurs between the baseline CT scan, showing no hemorrhage, and a follow-up CTscan showing new hemorrhage, consistent with new or worsening symptoms and signs. A serious systemic hemorrhage is defined as a bleeding episode other than ICH that is considered life-threatening by the investigator or that results in a drop in haemoglobin concentration greater than or equal to $5 \mathrm{~g} / \mathrm{dLor}$ requires two or more units of packed red blood cell transfusion. Orolingual angioedema is defined as localised swelling of the tongue, lips or oropharynx temporally related to the onset of alteplase infusion by less than six hours. ${ }^{14}$ Any other serious adverse event is defined as one that is life-threatening, permanently disabling, or sufficiently incapacitating such that the patient requires prolonged hospitalisation or requires prescription drug therapy.

Secondary outcome measures include discharge mRS score, discharge disposition, place of residence at 90 days, length of stay, asymptomatic ICH, and all ICH. Importantly, because the natural history of ischemic stroke frequently includes hemorrhagic conversion, ${ }^{15}$ asymptomatic ICH is not being collected as a serious adverse event.

\section{Patient follow-up}

All patients are followed for 90 days from stroke onset. The majority of patients were examined in person; where this has proved impossible, phone follow-up has been undertaken. Patient outcome assessments are not blinded.

\section{Data collection and management}

A standard single page data collection form was designed and has been modified once since the outset of the study. Simple additional forms to capture treatment, serious adverse events, CT scan scores and three-month outcome are used. All of these forms were printed and distributed to registered centres. In addition, a website ${ }^{16}$ was established to facilitate easy distribution of information. All data collection forms are available, in English and French, for printing directly off our website at www.strokeconsortium.ca/CASES.

Baseline demographic characteristics, stroke characteristics and severity, interval times, baseline blood tests and stroke risk factors are all collected. Outcome measures at discharge and at 90 days are assessed. Each investigator has been asked to grade the baseline and follow-up brain CT scan according to a validated scoring system, Alberta Stroke Program Early CT (ASPECTS), developed in Calgary and Houston. ${ }^{17}$ Each centre has been asked to make copies of baseline, 24h (and where possible) follow-up brain CT scans to send to the data collection centre for review.

All definitions were provided to study centres in a protocol binder. All the data are being collated on a custom database using Access97 (Microsoft Corp, Seattle, Washington). Data are being analysed using Intercooled STATA 6.0 (Stata Corp, College Station, Texas).

\section{Central adjudication of CT scans}

All CT scans are centrally reviewed by a group composed of two stroke neurologists and one neuroradiologist using a formal, standardised protocol. Baseline scans will be reviewed for potential protocol violations such as subtle hemorrhage and then scored using the ASPECT score, with knowledge of the side of the patient's symptoms. Each rater will score the scan independently, followed by a consensus score. The majority (two of three) will rule in instances of disagreement for the group score. Twenty-four hour scans will be coded for hemorrhage according to the ECASS criteria ${ }^{2}$ as well as re-scored using the ASPECT scoring system. In each case the consensus score will be the final score.

\section{Data validity}

The internal validity of the data will be assessed. At the data centre, the database will be manually cross-checked against every data entry form for accuracy. An ongoing Ontario stroke registry pilot study database will be used to cross-check information at three Ontario sites. Four sites (two community, two tertiary) have been randomly selected and will undergo an onsite audit by chart review of entered patients. At those sites, pharmacy records will be used to track alteplase usage during the study period and confirm that at that site all stroke patients treated with alteplase have been entered into CASES.

External validity of the data will be assessed by survey methodology. Each hospital in Canada that has an on-site CT scanner and is not registered in CASES will be contacted by phone and by mail. The survey will focus upon the departments of pharmacy, internal medicine, neurology (if applicable) and emergency medicine. The total number of stroke patients treated with alteplase and the total number of patients who suffered symptomatic ICH will be sought.

\section{Hypotheses and statistical analysis}

\section{Safety}

The proportions of patients suffering any safety outcome will be reported in frequency tables with exact binomial confidence intervals. 


\section{Effectiveness}

Effectiveness will be assessed by examining the proportion of patients achieving a good outcome, defined as an $\mathrm{mRS} \leq 2$ at 90 days.

\section{Predictors of outcome}

A multivariable logistic regression model will be developed to identify independent predictors of good outcome. A priori, the following seven variables will be tested in the model as predictors of outcome: baseline NIHSS score, baseline ASPECT score, age, baseline serum glucose, baseline mean arterial pressure, onset-to-treatment time, on-protocol treatment (appropriateness). A univariate analysis of all baseline variables as predictors of outcome will be conducted. For maximum sensitivity, any variable that is significant at $\alpha<0.5$ will be additionally considered for inclusion in the final model. ${ }^{18}$ Only variables which reach conventional significance at $\alpha \leq 0.05$ will be retained in the final model.

\section{ASPECTS}

Receiver-operator curve methodology will be used to assess the ASPECT score as predictor of outcome. A cut-off score will be defined that provides maximum sensitivity and specificity. This will permit dichotomization of scores to be examined as a predictor of good outcome using Fisher's exact test.

Pre-specified secondary analyses will include the examination of interval times, length of stay, the effect of treatment experience (number of patients treated per unit time) at high volume vs. low volume centres and over the time course of the study. No formal comparisons will be made to historical data; however, the provision of confidence intervals surrounding our estimates will allow indirect comparison.

\section{Sample Size}

The projected sample size of 1100 patients is a sample of convenience. With an estimated $(n=61)$ symptomatic intracerebral hemorrhages, this will provide $95 \%$ confidence intervals as follows:

SICH rate: $5.5 \%(4.3-7.1)$

Similarly, the number of patients who have an excellent neurological outcome $(n=330)$ will have the associated $95 \%$ confidence interval as follows:

Good outcome rate: $30.0 \%$ (27.3-32.8)

Estimating sample size for logistic regression is problematic but the sample size should also be large enough to allow clinically meaningful precision in the estimates of the magnitude of effect of predictor variables.

\section{Conduct of the trial and ethical considerations}

The CASES study committee has a weekly minuted review meeting. Organisational and communication issues and other ongoing problems are reviewed. All new cases entered into the study are reviewed and any serious adverse events are presented for assessment. Serious adverse events related to alteplase are followed up with a phone call to the local study investigator to try to explore the circumstances surrounding that event.

All data are considered confidential and anonymous. Patients are numbered sequentially by centre, and patient identifiers (eg. name, hospital number) are kept locally but not at the data centre. As a matter of safety, Hoffmann-La Roche Canada Ltd. is being notified of serious adverse events when they occur and these events are incorporated into the global safety database for alteplase. Information sent to Hoffmann-La Roche Canada Ltd., is completely blinded to patient and study centre. Any release of information is discussed at the weekly CASES meeting prior to dissemination.

Informed consent for submission of data may be required, and physician-investigators have been asked to submit the data collection protocol to the local institutional research and ethics review board. There is a significant variability in the requirement for informed consent by centre.

\section{Progress}

A total of 76 centres have been registered. Of these, 60 are actively submitting data to the study. There are 25 tertiary care sites and 35 community sites. Centres from nine of the 10 provinces in Canada are represented. A total of 944 patients have been entered. Recruitment progress is shown in Figure 2.

Preliminary results have been published in abstract and summary form. ${ }^{19,20}$ In brief, there are some differences between the patient populations treated in Canada and that published by the NINDS investigators (Table). Importantly, the severity of stroke has been similar to that previously observed. However, the symptomatic hemorrhage rate is $4.7 \%$ (3.4-6.2). Follow-up information is available for $61 \%$ of patients and outcomes have been entirely analogous to those observed in the NINDS tPA Stroke Trial.

The extent of follow-up and completeness of both clinical and CT data will only be known at the study's conclusion. We anticipate that greater than $90 \%$ data completeness will be achieved. Because adverse events have been routinely reported within a week of the event, we are confident that the quoted rate of symptomatic ICH is accurate. However, it should be noted

Table: Baseline Demographic and Pre-morbid Patient Characteristics vs. NINDS tPA Stroke Trial

\begin{tabular}{lll}
\hline & CASES (n=812) & NINDS (n=312) \\
Gender & $54 \%$ male & $58 \%$ male \\
Handedness & $96 \%$ RH & --- \\
Symptom side & $55 \%$ right side of body & --- \\
Median age & 70 (mean) & 67 (mean) \\
Ethnicity & $93 \%$ caucasian & $65 \%$ caucasian \\
NIHSS score & 14 (median) & 14 \\
Hypertension & $48 *$ & 67 \\
Prior TIAor stroke & $22 *$ & 31 \\
Atrial fibrillation & 22 & 21 \\
Anti-platelet therapy & $21 *$ & 40 \\
Current smoker & $16 *$ & 34 \\
Diabetes & $15 \% *$ & $22 \%$ \\
Congestive heart failure & $6 \% *$ & $15 \%$ \\
Valvular heart disease & $4 \%$ & $5 \%$ \\
\end{tabular}

$* \mathrm{p}<0.05$ 


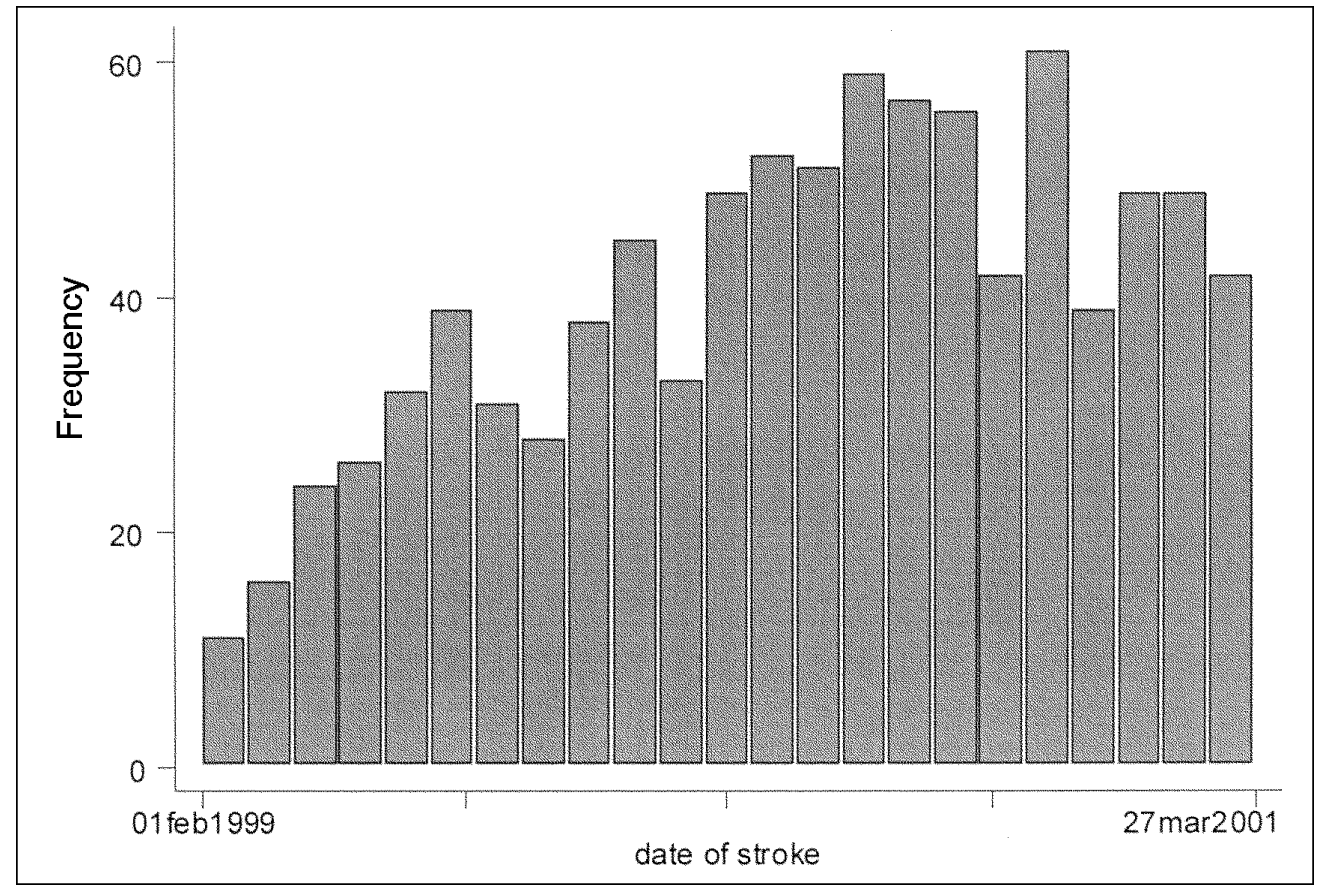

Figure 2: Recruitment profile

that the study does not have complete population data, and it is therefore likely that some cases of ICH have occurred in centres not reporting to the study, making the assessment a slightly biased underestimate of the true incidence.

Across the country, there has been a variation by centre in the role of Institutional Review Board review and approval. Larger academic centres have established committees and review has been conducted with varying stipulations. For example, some centres have been disallowed from submitting patient initials or postal code. Some small community hospitals do not have Ethics Review Committees established and consequently the CASES protocol has been reviewed by the local Medical Advisory Council.

\section{Conclusions}

This paper outlines the development and methods for the CASES study. CASES is a prospective registry of intravenous alteplase therapy for AIS that is nearing completion, with final results expected in early 2002. It provides a structured way of introducing a complex therapy into clinical practice in Canada, and we anticipate that it will provide new insights into the management of AIS. It represents a collaboration among multiple stakeholders - physicians, industry, federal regulators, and a charitable organisation - to further the treatment and care of stroke patients.

The early results are encouraging. Canadian AIS patients have been treated safely with alteplase with a symptomatic hemorrhage rate $-4.7 \%$ - that is entirely consistent with, if not better than, that observed in randomised trials. This suggests that Canadian physicians are applying this therapy judiciously.

We caution that the risks of hemorrhage and benefit demonstrated in randomised trials are highly dependent upon closely following recommended guidelines for alteplase use; the therapeutic index is narrower than alteplase for acute myocardial infarction. ${ }^{21}$ CASES is functioning as an additional safety measure by ensuring that physicians stick to the accepted protocol.

CASES will attempt to provide an assessment of the effectiveness of thrombolysis in stroke. Effectiveness may be thought of as a quality control issue. Many medical treatments have demonstrated efficacy in the strict, tightly controlled arena of randomised clinical trials but this may not necessarily translate into effectiveness in the community. ${ }^{22}$ Initial experience in Calgary and elsewhere, ${ }^{21,23-25}$ has demonstrated that protocol violation, which may occur both intentionally and unwittingly, as new treatments are used, substantially increases the risk of symptomatic hemorrhage and poor outcome. However, experienced US centres have reported comparable postmarketing outcomes to the NINDS trial in terms of three month outcome and risk of ICH. ${ }^{26}$ CASES will assess the effectiveness of alteplase therapy for AIS in the Canadian context using a number of outcomes (stroke scale scores, mortality, symptomatic hemorrhage rates and high volume vs. low volume centres).

CASES will function as part of a larger quality assurance loop by providing: (1) safety information to allow the final licensure of alteplase for the treatment of AIS, (2) further information on the types of patients who most benefit from this therapy allowing progressive modification of clinical algorithms and, (3) information on patients who are not being treated that may be used for planning stroke care strategies. The treatment of AIS is a complex health technology for which diffusion into the community is aided by the backbone structure provided by a formal study. ${ }^{27,28}$ 


\section{ACKNOWLEDGEMENTS}

Dr. Hill is funded by grants from the Canadian Institutes for Health Research, the Heart and Stroke Foundation of Canada, and the Alberta Heritage Foundation for Medical Research.

Dr. Buchan is funded by grants from the Heart and Stroke Foundation of Alberta/NWT, and the Alberta Heritage Foundation for Medical Research.

\section{ApPendix 1: The CASES Collaborators}

\section{A. The CASES Investigators}

Alberta: Alastair M Buchan (Principal Investigator), Michael D Hill (Co-principal investigator), Andrew M Demchuk, Keith M Hoyte, Gary M Klein, Philip A Barber, Nancy Newcommon, Andrea Cole-Haskayne, Zeenie Ramji, Diana Czechowsky, Ashfaq Shuaib, Edina Kadribasic, Toni Winder, Carolyn Walker, Prafull Parekh, Scott Wilson, Yolande Westra, Robert Burris, Gail Deagle, Laurel Reynolds

British Columbia: Philip Teal, Andrew Woolfenden, Claire Johnston, Todd Collier, Judy Moa, Donald Cameron, Loree Tadey, Cate Earl, Tracy Sacre, Kennely Ho, Frank Kemble, Elizabeth Mauthe, Lynda MacFarlane, David Novak, Richard Grosch, Joe Haegart, Cathy Metcalfe, Milton Wong, Barbara Boychuk, Terry Curran, Suzanne Bailey, Anthony Costantino, Helen Costantino, David Craig, Michelle Mantle, Judith Huff, Michael Kenyon, Cutler, Chuck Mahoney, Jo Thoburn

Manitoba: Brian A Anderson, Mohammad Nagaria, Dan F Gladish, Brian Schmidt, Douglas Eggertson, Douglas Hobson, Lawrence Hudson, Greg McGinn, Farid Esfahani

New Brunswick: Peter Bailey, Gregg MacLean, A MacDougall, Peggy Cook, Shelly Alward, Mary Freeman-McCraek, Lynn Reid, Byrne Harper

Newfoundland: Alan Goodridge, William Pryse-Phillips, Mark Stefanelli, Jim Scott

Nova Scotia: Stephen Phillips, Gordon Gubitz, Judith Jarrett, Richard Leckey, G Maharaj

Ontario: Rudolph Arts, Sylvia Styling, Vladimir Hachinski, J David Spence, Brian Silver, Bart M Demaerschalk, Ashok Devasenapathy, Jose G Merino, Edward H Wong, J Arturo Tamayo Mendoza, Blaine Foell, Fali Poncha, Connie A Frank, Mary M McTaggart, Frank Silver, Cheryl Jaigobin, Shelley Yantha, Pamela Urzua, Relu Wiegner, Bob Duke, Wes Oczkowski, David Fletcher, Sera Nicosia, Nancy Pyette, Sandy Black, Na Jiang, David Gladstone, Edwin Klimek, Chidambaram Yegappan, Marianne Klimek, Jeanine Robinson, Daniel Selchen, Alnoor Dhanani, Bryan Temple, Heather Hink, Deidre Davidson, Kathryn Leblanc, Suzanne Christie, Lucian Sitwell, Andre Douen, Antoine Hakim, Nicole Pageau, Hyman Rabinovitch, Martin Del Campo, Neville Bayer, David Morgenthau, Gabrielle De Veber, Andrew Kertesz, Darlyne Morlog, Richard Magder, Garry Moddel, Chidambaram Yegappan, Iram Zando, Martin Lees, Peter Stys, Betty Anne Schwarz, David Howse, Richard Riopelle, Cindy Bolton, Sandy Weatherby, Dianne Groll, Mukul Sharma, Hiren Desai, Michael Winger, Dave Tamblyn, Stephane Sauve, Hui Lee, Deon Leow, Dwight Stewart, Susan Fawcett, Vince dePaul,

Quebec: Robert Cote, Liam Durcan, Anne-Marie Fontaine, Lisa
Wadup, Francois Jacques, Denis Halle, André Bellavance, Leo Berger, Linda Moisan, Jeanne Teitelbaum, Nicole Lachance, Ariane Mackey, Denis Simard, Annette Hache, Barbara Léger, Lison Fournier, Sophie Dubé, Jeffrey Minuk, Louise-Hélène Lebrun, Andre Durocher, Nicole Daneault, Sylvain Lanthier, Marlene Lapierre, Marie-Paul Desrochers, Michel Beaudry, Doris Boivin, Donald Rivest, Marc Petitclerc, Marie Berberl, Charles Beaurivage, Albert Lamontagne, Serge Desviens

Saskatchewan: Ali Rajput, Christopher Voll, Michele Rajput, Aileen Schultz, Felix Veloso, Barbara Seal, L Moodley

\section{B. Hoffmann-La Roche Representatives}

Alberta: Alanna Campbell, Frank Heron

British Columbia: Kath Davy

Manitoba/Saskatchewan: Kath Davy

Ontario: Angie McAllister

Quebec: Rene Garneau

Atlantic Canada: Linda Mosher

Administrative Asst: Vivian Thompson

Regional Business Manager: Jean-Rene Poirier

Associate Medical Directors: Kate Lawrence, Esther Tan

Director of Marketing, Hospital Care : Alan LeVoguer

\section{Heart and Stroke Foundation of Canada} Elinor Wilson

\section{Canadian Stroke Consortium}

President: Philip Teal

Past President: John Norris

Staff: Donna Huber

\section{REFERENCES}

1. NINDS tPA Stroke Study Group. Tissue plasminogen activator for acute ischemic stroke. New Eng J Med 1996; 333: 1581-1587.

2. European Cooperative Acute Stroke Study (ECASS): Intravenous thrombolysis with tPA for acute hemispheric stroke. JAMA1995; 274: 1017-1025.

3. Hacke W, Kaste M, Fieschi C, et al for the ECASS-2 Investigators. Randomised double-blind placebo-controlled trial of thrombolytic therapy with intravenous alteplase in acute ischemic stroke (ECASS-2). Lancet 1998; 352: 1245-1251.

4. Clark WM, Wiseman S, Albers GW, et al for the ATLANTIS Investigators. Recombinant tissue-type plasminogen activator for ischemic stroke within 3-5h of symptom onset. JAMA1999; 282: 2019-2026.

5. Grotta J. Should thrombolytic therapy be the first-line treatment for acute ischemic stroke? t-PA - the best current option for most patients. N Engl J Med 1997;337(18):1310-1313.

6. Caplan LR, Mohr JP, Kistler JP, Koroshetz W. Should thrombolytic therapy be the first-line treatment for acute ischemic stroke? Thrombolysis - not a panacea for ischemic stroke. N Engl J Med 1997;337(18):1309-1310; discussion 1313.

7. Hachinski V. Thrombolysis in stroke: between the promise and the peril. JAMA1996;276(12):995-996.

8. Hoffman JR. Predicted impact of intravenous thrombolysis. Another trial is needed. Br Med J 2000; 320:1007.

9. Hoffman JR. Should physicians give tPA to patients with acute ischemic stroke? Against: and just what is the emperor of stroke wearing? West J Med 2000;173:149-150.

10. Wardlaw JM, del Zoppo G, Yamaguchi T. Thrombolysis for acute ischemic stroke. The Cochrane Library 1999 Issue 4.

11. Anonymous. Canadian Adverse Drug Reaction Newsletter: Alteplase (Activase rt-PA) for acute ischemic stroke: special precautions for new indication. CMAJ 1999;161:73-74. 
12. Anonymous. The Changing Face of Heart Disease and Stroke in Canada 2000. Heart and Stroke Foundation of Canada. Ottawa, Canada 1999.

13. Norris JW, Buchan A, Cote R, et al for the Canadian Stroke Consortium. Canadian guidelines for intravenous thrombolytic treatment of acute stroke. Can J Neurol Sci 1998;25:257-259.

14. Hill MD, Barber PA, Takahashi JL, et al. Anaphylactoid reactions and angioedema during alteplase treatment of acute ischemic stroke. Can Med Assc J 2000;162:1281-1284.

15. Hornig CR, Bauer T, Simon C, Trittmacher S, Dorndorf W. Hemorrhagic transformation in cardioembolic cerebral infarction. Stroke 1993;24:465-468.

16. http://www.strokeconsortium.ca/CASES/.

17. Demchuk AM, Karbalai H, Grotta JC, Buchan AM. Early CT scoring system predicts hemorrhage and outcome after intravenous thrombolytic therapy (abstract). Stroke 1999;30:248.

18. Teyerberg EW, Eijkemans MJC, Habbema JDF. Stepwise selection in small data sets: a simulation study of bias in logistic regression analysis. J Clin Epidemiol 1999;52:935-942.

19. Hill MD, Woolfenden A, Teal P, Buchan AM. Intravenous alteplase for acute ischemic stroke: the Canadian Experience. The Stroke Interventionalist 2000;2:5-7.

20. Hill MD, Buchan AM for the CASES Investigators. The Canadian Activase for Stroke Effectiveness Study (CASES): Interim results (Abstract). Stroke 2001;32:323.
21. Buchan AM, Barber PA, Newcommon N, et al. Effectiveness of tPA in acute ischemic stroke: outcome relates to appropriateness. Neurology 2000;54:679-684.

22. Rochon PA, Anderson GM, Tu JV, et al. Use of ß-blocker therapy in older patients after acute myocardial infarction in Ontario. CMAJ 1999;161:1403-1408.

23. Grond M, Stenzel C, Schmulling S, et al. Early intravenous thrombolysis for acute ischemic stroke in a community-based approach. Stroke 1998;29(8):1544-1549.

24. Tanne D, Bates VE, Verro P, et al. Initial clinical experience with IV tissue plasminogen activator for acute ischemic stroke: a multicenter survey. The t-PA Stroke Survey Group. Neurology 1999; 53(2):424-427.

25. Albers GW, Bates VE, Clark WM, et al. Intravenous tissue-type plasminogen activator for treatment of acute stroke. JAMA 2000;283:1145-1150.

26. Chiu D, Krieger D, Villar-Cordova C, et al. Intravenous tissue plasminogen activator for acute ischemic stroke: feasibility, safety, and efficacy in the first year of clinical practice. Stroke 1998;29(1):18-22.

27. Battista RN. Innovation and diffusion of health-related technologies. Int J Technol Assess Health Care 1989;5:227-248.

28. Weingart SN. Acquiring advanced technology: decision-making strategies at twelve medical centres. Int J Technol Assess Health Care 1993;9:530-538 